\title{
Combination of Various Weighting Functions to Improve Modal Curve Fitting for Actual Mechanical Systems*
}

\author{
Meifal RUSLI**, Masaaki OKUMA ${ }^{* *}$ and Takeshi NAKAHARA**
}

\begin{abstract}
Modal curve fitting methods on frequency domain are based on the least square method to estimate the modal parameters from frequency response functions (FRFs in brief) of test structures. In order to minimize the least square errors between experimental and estimated FRFs, several kinds of weighting functions were proposed and widely applied in practical use. However, using the same weighting function in modal curve fitting for a set FRFs of test structure often makes the results of the FRFs of some measurement points be fitted with good accuracy but other ones be not. In this paper, the authors propose the combination use of various kinds of weighting functions in least square process of modal curve fitting methods to deal with the measurement data of actual and complex mechanical systems. This method automatically selects and applies the most appropriate weighting function to the FRF of each measurement point by comparing the minimum total relative mean square error (RMSE). The case study shows that the proposed idea allows the modal curve fitting method puts better curve fitting results out so that more accurate mathematical model will be obtained.
\end{abstract}

Key Words: Linear Least Square, Nonlinear Least Square, Curve Fitting, Weighting Function

\section{Introduction}

Due to the increasing of complexity of modern mechanical structures and strong demand for high quality of dynamics characteristics, accurate mathematical modeling has become a necessity for successful design. Over the past three decades many researchers have devoted their effort to develop the technique aimed at producing reliable identification of the dynamics properties of mechanical structures.

In frequency domain, a number of algorithms have been developed to estimate modal parameters from measured frequency response or impulse response data ${ }^{(1),(2)}$. However, such algorithms cannot answer the question of added value with respect to the already existing method ${ }^{(3)}$. That is why the comparison of one algorithm to another has become one of the trusted current researches today.

The least square method is the mathematical base of the modal curve fitting methods using frequency response

* Received 4th August, 2005 (No. 05-4128)

** Department of Mechanical and Aerospace Engineering, Tokyo Institute of Technology, 2-12-1-I3-15 Ookayama, Meguro-ku, Tokyo 152-8552, Japan.

E-mail:meifal@stu.mech.titech.ac.jp;

mokuma@mech.titech.ac.jp data. Least square method (LS method in brief below) is based on minimizing the error norm between measured Frequency Response Function (FRF) and the associated estimation model FRF.

To improve the capability of the least square algorithm, several kinds of weighting functions were proposed $^{(4)-(11)}$. In practical use, only a kind of weighting function among them is applied to a set of FRFs of a test structure. However, it shows the inconsistence, bad quality and inefficiency in the case of increasing the complexity of test structures. In fact, complex structures tend to have big and variant noise contribution at measurement points and to raise the value of the error ${ }^{(4)}$. Having seen the inconsistence, structure complexity, and noise, it is ineffective to apply only single weighting function in complex mechanical system modeling for overall measurement points of SIMO or MIMO system.

In this paper, the authors propose the implementation of the combination of various kinds of weighting functions for the least square based modal curve fitting methods. The most appropriate weighting function is applied to the FRF of each measurement point by being selected among various kinds of weighting functions.

There are two cases are presented here using 9 kinds of weighting functions. The first case is a simple beam, 
and the second is 3-cylinder combustion engine. Those weighting functions are divided into weighted nonlinear least square (NLS) and weighted linear least square (LS). On the first step, the computation of curve fitting of FRFs is conducted using all weighting functions separately for all measurement points. On the second step, the best weighting function for each measurement point is determined by comparing relative square error norms (RMSE) with respect to the weighting functions. Each measurement point may take different best weighting function. The final step is to compute curve fitting using the combination of the best weighting functions for all measurement points.

\section{Nomenclature}

$A_{0}, A_{1}, \ldots, A_{m}:$ Constant

$B_{0}, B_{1}, \ldots, B_{n}$ : Constant

$D$ : Denominator of estimated FRF

$N$ : Numerator of estimated FRF

$E:$ Error

$F:$ Number of frequency points

$H:$ Transfer function

$\hat{H}$ : Estimated FRF

$H_{m}$ : Measured FRF

$W, W^{\prime}$ : Weighting Function

$\gamma^{2}$ : Coherence function

$\lambda$ : Error function

$\omega$ : Angular frequency

\section{Weighting Function in Modal Curve fitting}

The measured frequency response functions (FRFs) of linear-time invariant (LTI) and continuous-time systems can generally be modeled as the ratio of two frequencydependent polynomials as

$$
H(j \omega)=\frac{A_{0}+A_{1}(j \omega)+\cdots+A_{m}(j \omega)^{m}}{B_{0}+B_{1}(j \omega)+\cdots+B_{n}(j \omega)^{n}}
$$

where $m<n$. The measured FRF at discrete fourier transform between input and output is then modeled as

$$
\hat{H}\left(\omega_{f}\right)=\frac{N\left(\omega_{f}\right)}{D\left(\omega_{f}\right)}
$$

Based on the modal parameter estimation algorithms used today, there are many types of algorithms that frequently used. One of them is the least square method to minimize the squared error norm between measured data and the estimation model ${ }^{(1)}$. Some application of the least square will be discussed below.

\subsection{Weighted nonlinear least square (NLS)}

The objective function to be minimized in the least square based methods is written as.

$$
\lambda_{\mathrm{NLS}}=E_{\mathrm{NLS}}^{T} E_{\mathrm{NLS}}=\sum_{i=1}^{F}\left|H_{m}\left(\omega_{i}\right)-\hat{H}\left(\omega_{i}\right)\right|^{2}
$$

Minimization of this nonlinear least square equation (NLS) can estimate the coefficients of model expressed in
Eq. (2). If the FRFs are calculated using noisy output and input, the estimator is inconsistent from the formal point of view about the NLS. However, if one takes a precaution to avoid a division by zero, one may say that NLS estimator is "practically" consistent ${ }^{(4)}$.

Ewins $^{(5)}$ suggested attaching weighting factor to each frequency points of interest; hence curve-fitting process has to minimize such the total error using

$$
\lambda_{\mathrm{NLS}}=\sum_{i=1}^{F} W^{2}\left(\omega_{i}\right)\left|H_{m}\left(\omega_{i}\right)-\hat{H}\left(\omega_{i}\right)\right|^{2}
$$

where $W\left(\omega_{i}\right)$ is a proper weighting function.

Okuma $^{(6),(7)}$ then proposed to use a proper weighting function based on the maximum likelihood method. This function was dependent on measured coherence functions and FRFs. Firstly, Okuma proposed the weighting function below

$$
W_{\mathrm{OKM} 1}\left(\omega_{i}\right)=\frac{\gamma^{2}\left(\omega_{i}\right)}{1-\gamma^{2}\left(\omega_{i}\right)} \frac{1}{\left|H_{m}\left(\omega_{i}\right)\right|^{2}}
$$

According to application studies, Okuma later proposed the weighting function below as an alternative choice.

$$
W_{\mathrm{OKM} 2}\left(\omega_{i}\right)=\frac{\gamma^{2}\left(\omega_{i}\right)}{1-\gamma^{2}\left(\omega_{i}\right)} \frac{1}{\left|H_{m}\left(\omega_{i}\right)\right|}
$$

Both weighting functions can improve the NLS convergence. Minimizing error function can converge more rapidly than the primitive NLS.

\subsection{Weighted linear least square (LS)}

A linear least square approach requires model equations that are linear-in-the-parameters. The approximation was firstly presented for SISO system by Levi ${ }^{(8)}$. A nonlinear least square equation was reduced to a linear least square by multiplying equation error by the denominator of estimated FRF as the equation below.

$$
\lambda_{\text {LEVI }}=\sum_{i=1}^{F}\left|H_{m}\left(\omega_{i}\right) D\left(\omega_{i}\right)-N\left(\omega_{i}\right)\right|^{2}
$$

Two major drawbacks of the linearization of nonlinear least square in Eq. (7) are the overemphasizing of high frequency error in Eq. (3) and the large dynamic range of numbers in normal equation ${ }^{(4)}$. But this non-iterative least square in Eq. (7) may result in poor low frequency fits and ill-conditioned normal equations for identification problems with a wide dynamic frequency range.

In order to overcome the lack of sensitivity in low frequency errors, some modifications of linear least square have been published using a weighting function in equation errors. The weighted linear least square then developed in the following equation:

$$
\lambda_{\text {IWLS }}=\sum_{i=1}^{F} W^{2}\left(\omega_{i}\right)\left|H_{m}\left(\omega_{i}\right) D\left(\omega_{i}\right)-N\left(\omega_{i}\right)\right|^{2}
$$

where $W\left(\omega_{i}\right)$ is a proper weighting function. To compensate for the bias introduced in Levi scheme, Sanathanan 
and Koerner ${ }^{(9)}$ proposed an iterative procedure by using the weighting function below.

$$
W_{\mathrm{SAN}}=\frac{1}{\left|D\left(\omega_{i-1}\right)\right|_{\ell-1}}
$$

where subscript $\ell$ corresponds to the iteration number. The subsequent iterations tend to converge rapidly and the low frequency error can be reduced ${ }^{(4)}$.

Stroble proposed to minimize a relative error criterion instead of absolute criterion, by using the next function.

$$
W_{\text {STROBLE }}=\frac{1}{\left|H_{m}\left(\omega_{i}\right) D\left(\omega_{i}\right)\right|}
$$

The iteration is limited to one step $(i=1)$ and initial denominator coefficients $(i=0)$ are obtained by a prior analysis of the logarithmic amplitude response. t'Mannetje then modified to raise the weighting function in Eq. (9) to a certain power $r$, like the equation below.

$$
W_{\mathrm{t}^{\prime} \text { MANNETJE }}=\frac{1}{\left|D\left(\omega_{i}\right)\right|^{r}}, \quad r \in[0, \infty]
$$

Two special cases of Eq. (11) are the linear square method $(r=0)$ and Sanathanan and Koerner's method $(r=1)$. If Sanathanan and Koerner's method does not converge, the relaxation $(r<1)$ is helpful.

Whitfield $^{(10)}$ used periodic signal excitation assumption to develop weighting function by integral approach to reduce iteration step of Eq. (8). That is,

$$
W_{\text {INTEGRAL }}=\frac{1}{|j \omega|^{d}}
$$

The integral approach is used to overemphasize the low frequency errors. This is confirmed by the second-order simulation example where the integral approach results in a stable transfer function estimated by very large approximation error ${ }^{(4)}$.

Verboven $^{(11)}$ used a weighted linear square formulation for the various implementations by means of a nonparametric weighting function in Eq. (13) using FRF data.

$$
W^{2}\left(\omega_{i}\right)=\frac{\left|H_{m}\left(\omega_{i}\right)\right|}{\operatorname{var}\left(H_{m}\left(\omega_{i}\right)\right)}
$$

where possible correlations between the FRF are neglected. This non-parametric weighting function avoids the need for an iterative approach. And the variant of measured FRFs is

$$
\operatorname{var}\left(H_{m}\left(\omega_{i}\right)\right)=\frac{1}{M} \frac{\left(1-\gamma^{2}\left(\omega_{i}\right)\right)}{\gamma^{2}}\left|H_{m}^{2}\left(\omega_{i}\right)\right|^{2}
$$

where $M$ is number of averaging of measured FRF.

\subsection{The Combination of Weighted Least Square (CWLS)}

The Combination of weighted least square (CWLS) is proposed by selecting the best weighting function to curve fit FRF in each measurement point. Using this method the error of curve fitting can be minimized and more accurate modal parameters will be obtained. The CWLS works in three steps. The first step consists of computation of curve fitting of FRFs using all available weighting functions separately for all measurement points and calculation of the error of each weighting function in all measurement points. The next step is selecting the best weighting function in each point. The final step is applying the best weighting function in each point. Therefore CWLS will contain some kinds of weighting functions as the best weighting function in each measurement point.

To determine the error of all kinds of weighting functions in each measurement points in the first step, total error of curve fitting in one measurement point is calculated by total relative mean square error as Eq. (15) below

$$
\text { Total RMSE }=\sum_{i=1}^{F}\left|\frac{\left(\hat{H}\left(\omega_{i}\right)-H_{m}\left(\omega_{i}\right)\right)}{H_{m}\left(\omega_{i}\right)}\right|^{2}
$$

By comparing the total error of all weighting functions, the best and the most proper weighting function for each FRF of measurement point can be determined. Each FRF can use different kind of weighting function from one to another. The next step is to carry out the modal curve fitting again using an individually proper weighting function for FRFs of all measurement points.

The abovementioned of all kinds of weighting functions are applied to the case studies in this paper by using the modal parameter estimation program in the software made by Okuma ${ }^{(12)}$. To obtain a new model for each iteration step, the error $\boldsymbol{E}$ of natural angular frequency and damping ratio is calculated by using sensitivity analysis, like equation below.

$$
E=\left(T^{H} W T\right)^{-1} T^{H} W \Delta h
$$

where $\boldsymbol{T}$ is sensitivity matrix corresponding to natural angular frequency and damping ratio. $\boldsymbol{W}$ is weighting function, and $\Delta \boldsymbol{h}$ is the difference of estimated and measured FRF. This error equation is used to obtain the error function of each methods explained in section 2.1 and section 2.2

\section{Case Study}

\subsection{The first example}

The test structure of the first case is a simple uniformed beam under the free-free boundary condition. The results of modal parameter identification for it are compared among the three kinds of methods that use weighted NLS, weighted LS and the CWLS respectively. Hammer testing is conducted to get FRFs. The number of measurement points is 11 on the beam. The frequency range of FRF measurement is $5-180 \mathrm{~Hz}$. FRFs and coherence functions are obtained using 4-channel FFT analyzer.

Firstly, measured FRF data are curve fitted using weighted NLS and weighted LS. Figure 1 shows curve fitting results of two measurement points by using all 


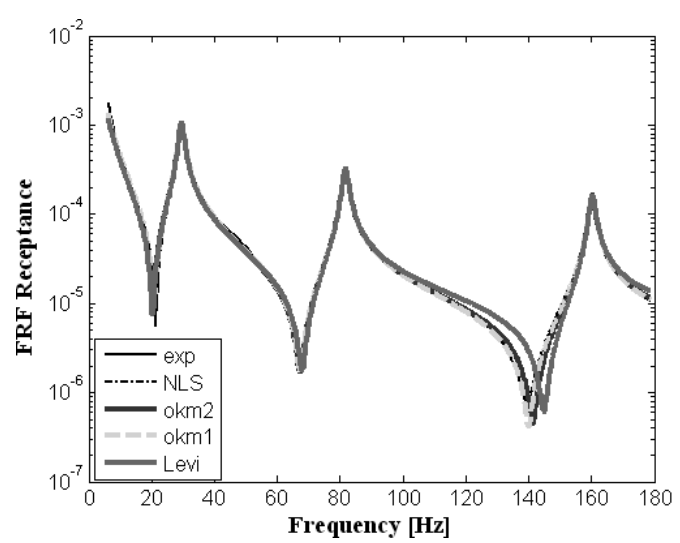

(a)

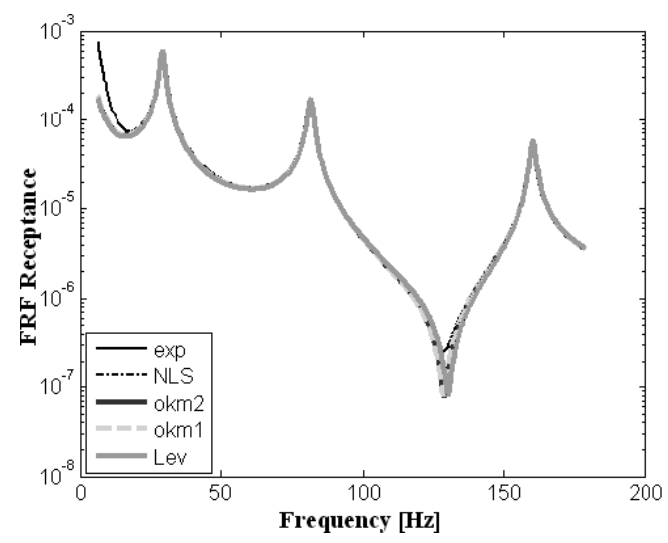

(c)

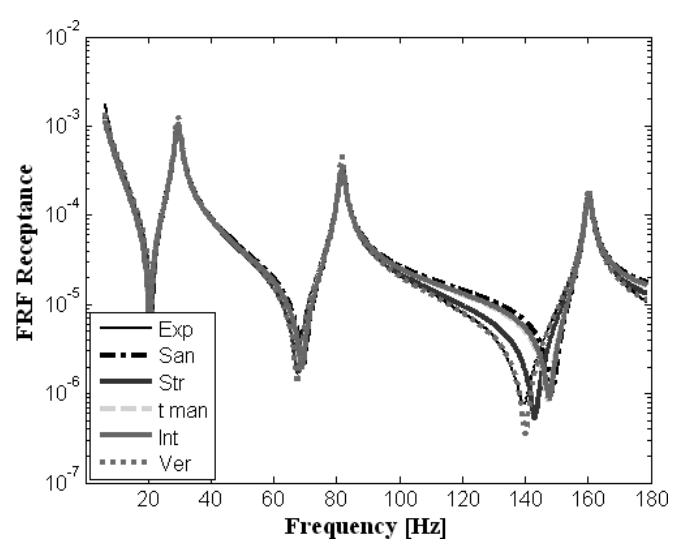

(b)

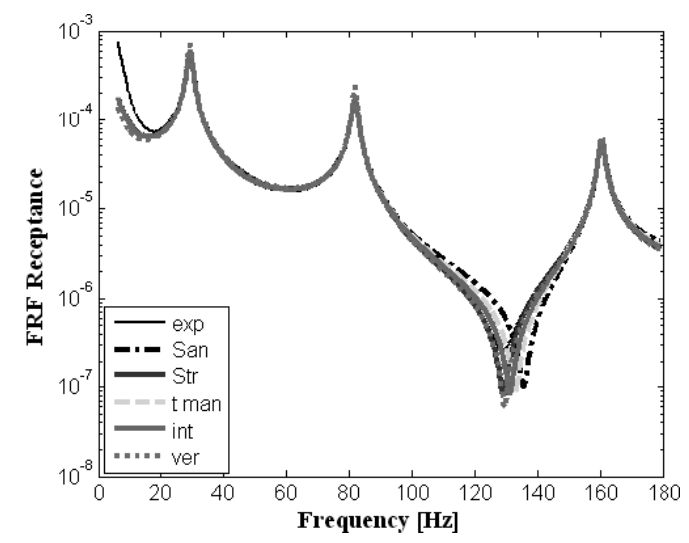

(d)

Fig. 1 Curve fit using all weighting functions for measurement point number $1(a, b)$ and measurement point number 7 (c, d)
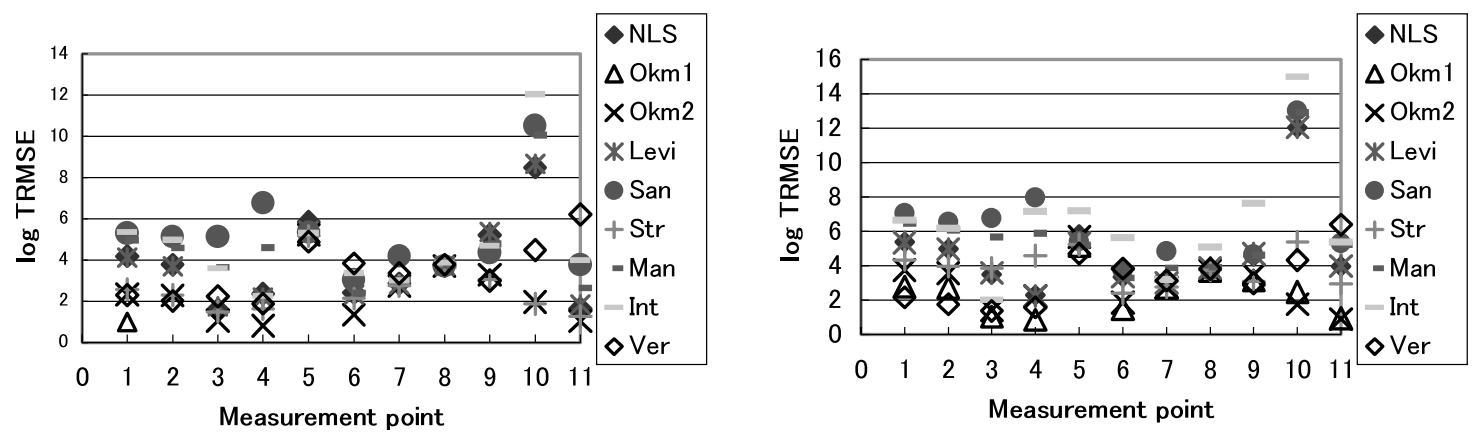

Fig. 2 Total RMSE in each measurement points using original weighting function (left) and Total RMSE for frequency scale weighting function (right)

weighting functions. It shows that every weighting function makes good curve fitting at resonant peaks but ill condition at anti-resonant.

Total RMSE in all cases of using different weighting functions for FRFs of every measurement point is presented in Fig. 2. It can be seen that there is no dominant weighting function that has the least total RMSE for all measurement points. A weighting function is good in one measurement point but may become worse in others. CWLS to be proposed in this paper applies the most appropriate weighting function to FRFs of each mea- surement point, according to the least total RMSE of all weighted NLS and Weighted LS. Figure 3 shows the number of measurement points for which each weighting function gets the least RMSE. It shows that weighting function Okuma-1 is the best for 5 measurement points, Okuma-2 for 2 measurement points and Verboven for 4 measurement points. Furthermore, CWLS automatically will choose Okuma- 1 for 5 points, Okuma- 2 for 2 points, and Verboven for 4 points. It will make CWLS be the best in a total 11 points (all points).

In order to improve the ill condition in all the fre- 

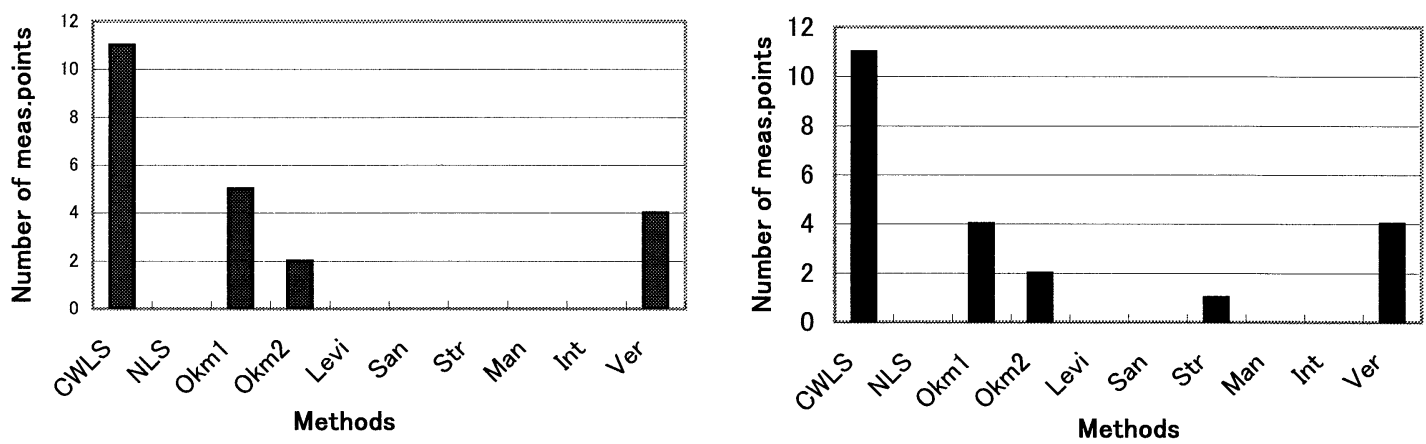

Fig. 3 The number of measurement points for which each weighting function gets the least RMSE, original weighting function (left) and weighting function with frequency scale (right)

Table 1 Weighting function that used in each measurement point in CWLS with original weighting function and frequency scale

\begin{tabular}{|c|c|c|c|c|c|}
\hline \multirow{2}{*}{$\begin{array}{c}\text { Meas. } \\
\text { Point }\end{array}$} & \multicolumn{2}{|c|}{ Original } & \multicolumn{2}{c|}{ frequency scale } & \multirow{2}{*}{} \\
\cline { 2 - 5 } & $\log$ (TRMSE) & Method & $\log$ (TRMSE) & Method & Improvement \\
\hline 1 & 2.1679 & Verboven & 2.3089 & Verboven & $(-)$ \\
\hline 2 & 1.7618 & Verboven & 2.0235 & Verboven & $(-)$ \\
\hline 3 & 1.048 & Okuma1 & 1.0633 & Okuma1 & $(-)$ \\
\hline 4 & 0.854 & Okuma1 & 0.8169 & Okuma1 & $(+)$ \\
\hline 5 & 4.7054 & Verboven & 4.8947 & Verboven & $(-)$ \\
\hline 6 & 1.4796 & Okuma1 & 1.3525 & Okuma1 & $(+)$ \\
\hline 7 & 2.7057 & Okuma1 & 2.7281 & Okuma2 & $(-)$ \\
\hline 8 & 3.7122 & Okumal & 3.716 & Okuma1 & $(-)$ \\
\hline 9 & 2.9859 & Verboven & 3.0014 & Verboven & $(-)$ \\
\hline 10 & 1.7623 & Okuma2 & 1.8812 & Stroble & $(-)$ \\
\hline 11 & 0.9028 & Okuma2 & 0.9534 & Okuma2 & $(-)$ \\
\hline
\end{tabular}

quency range of interest, normalization of weighting function using frequency scale is applied as

$$
W^{\prime}(\omega)=W(\omega) \omega
$$

When this frequency scale is used in weighting functions, the total RMSE is not affected significantly. Figure 2 presents that the least total RMSE in all measurement points are quite similar between with and without the frequency scale, and the number measurement points with the best condition in each weighting function is almost the same. When frequency scale of CWLS is applied, the total RMSE in each measurement point is not really improved as presented in Table 1. The sign of (+) in the last column of Table 1 means that application of frequency scale can reduce the total RSME of CWLS, and the sign of (-) means that application of frequency scale did not reduce the total RSME of CWLS in that point.

\subsection{The second example}

The second test structure is a 3-cylinder combustion engine with free-free boundary condition. All of inner components such as pistons, shaft, flywheel, and lubrication oil are installed. The experiment was conducted by impact hammer testing of a single point excitation in one direction. A tri-axial accelerometer is used to measure acceleration in three directions of 12 measurement points on the test engine in sequence. Totally, there are 36 spatial degrees of freedom of point measurements.
Table 2 Weighting function that used in each measurement point in CWLS with original weighting function and frequency scale (case study 2)

\begin{tabular}{|c|c|c|c|c|c|}
\hline \multirow[b]{2}{*}{ Meas. Point } & \multicolumn{2}{|c|}{ Original } & \multicolumn{2}{|c|}{ Frequency scale } & \multirow[b]{2}{*}{ Improvemen } \\
\hline & $\log$ (TRMSE) & Method & \begin{tabular}{|l|}
$\log$ (TRMSE) \\
\end{tabular} & Method & \\
\hline 1 & 4.1062 & Man & 4.0219 & Man & $(+)$ \\
\hline 2 & 4.1567 & Okuma2 & 4.3457 & Levi & $(-)$ \\
\hline 3 & 3.967 & Okumal & 3.878 & Man & $(+)$ \\
\hline 4 & 4.2965 & Okumal & 4.4713 & Okumal & $(-)$ \\
\hline 5 & 2.9302 & Okumal & 2.8476 & Verboven & $(+)$ \\
\hline 6 & 4.8242 & Okuma2 & 4.0974 & Man & $(+)$ \\
\hline 7 & 4.1977 & Man & 4.0445 & Man & $(+)$ \\
\hline 8 & 2.9299 & Okumal & 2.845 & Stroble & $(+)$ \\
\hline 9 & 4.5149 & Stroble & 4.3856 & NLS & $(+)$ \\
\hline 10 & 3.2095 & Man & 3.1875 & NLS & $(+)$ \\
\hline 11 & 3.7009 & Okumal & 3.7021 & Okumal & $(-)$ \\
\hline 12 & 3.4413 & Okuma2 & 3.4543 & Okumal & $(-)$ \\
\hline 13 & 4.3813 & Verboven & 4.5688 & Verboven & $(-)$ \\
\hline 14 & 5.1824 & Okumal & 5.2113 & Verboven & $(-)$ \\
\hline 15 & 2.6438 & Okumal & 2.6598 & Integral & $(-)$ \\
\hline 16 & 6.1481 & Verboven & 6.1763 & Verboven & $(-)$ \\
\hline 17 & 3.7814 & NLS & 3.553 & Man & $(+)$ \\
\hline 18 & 5.017 & Stroble & 5.108 & Stroble & $(-)$ \\
\hline 19 & 3.9101 & NLS & 3.9204 & Integral & $(-)$ \\
\hline 20 & 4.402 & Man & 4.7198 & Okumal & $(-)$ \\
\hline 21 & 3.9309 & Okumal & 4.0001 & Okuma2 & $(-)$ \\
\hline 22 & 3.9208 & NLS & 3.9223 & Integral & $(-)$ \\
\hline 23 & 5.2133 & Okumal & 5.1989 & Okumal & $(+)$ \\
\hline 24 & 3.7152 & Okumal & 3.7271 & Okumal & $(-)$ \\
\hline 25 & 4.3917 & Okumal & 4.4003 & Integral & $(+)$ \\
\hline 26 & 5.014 & Okuma1 & 4.9956 & Okuma1 & $(+)$ \\
\hline 27 & 3.6698 & Okumal & 3.7751 & Okumal & $(-)$ \\
\hline 28 & 6.5858 & Okumal & 6.549 & Okumal & $(+)$ \\
\hline 29 & 2.0165 & Okumal & 2.0586 & Okumal & $(-)$ \\
\hline 30 & 3.7118 & Okumal & 3.7582 & Okumal & $(-)$ \\
\hline 31 & 4.2307 & Man & 4.1855 & NLS & $(+)$ \\
\hline 32 & 3.714 & Verboven & 3.7353 & Verboven & $(-)$ \\
\hline 33 & 4.6071 & Levi & 4.6074 & Integral & $(-)$ \\
\hline 34 & 5.7076 & Okumal & 5.7685 & Okumal & $(-)$ \\
\hline 35 & 3.3134 & Okumal & 3.2865 & Okumal & $(+)$ \\
\hline 36 & 3.9246 & Okumal & 3.9363 & Okumal & $(-)$ \\
\hline
\end{tabular}

The number of sampling frequency points is 1024 from 0 until $800 \mathrm{~Hz}$. Recorded acceleration signal and position of accelerometer was used to find the dynamics parameters of the engine in which FRFs in lower frequencies than $40 \mathrm{~Hz}$ are cut off.

In the first step of curve fittings, each weighting function is applied to FRFs of all measurement points. Figure 4 shows the curve fitting result of using each weighting function for FRF of two measurement points; point FRF and a transfer FRF. The total RMSE of using each weighting function for all measurement points is shown in Fig. 5. It can be seen that some of weighting functions such as Sanathanan and Stroble tend to have ill condi- 


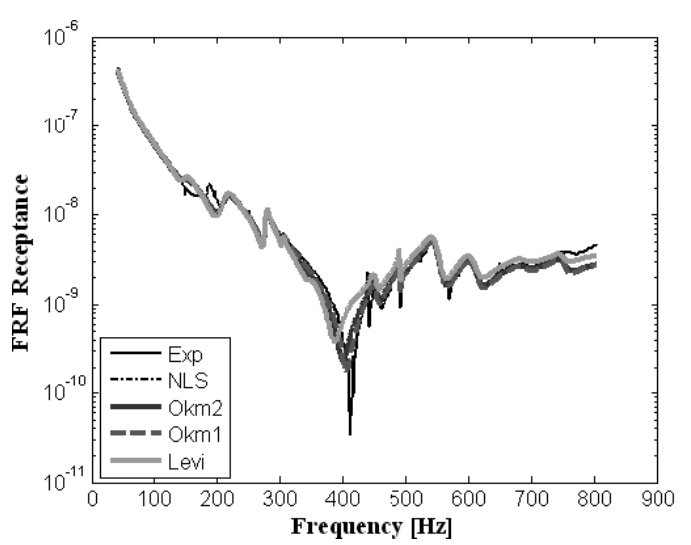

(a)

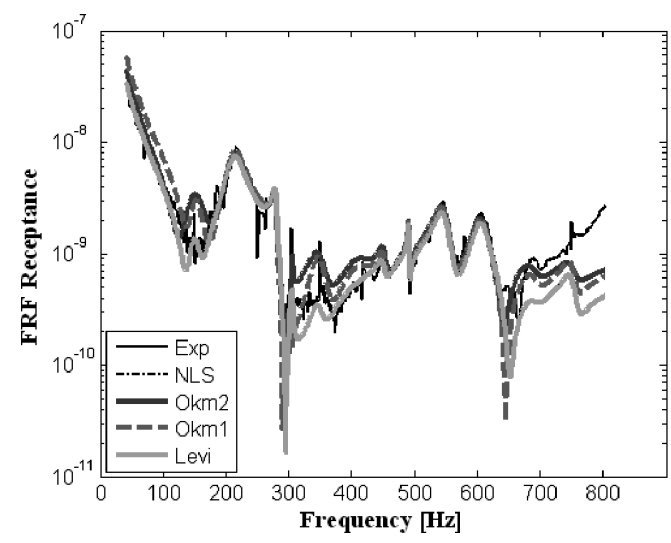

(c)

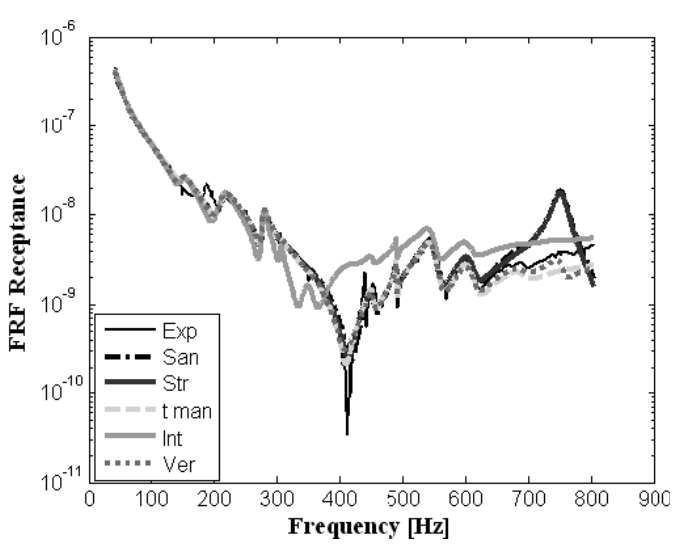

(b)

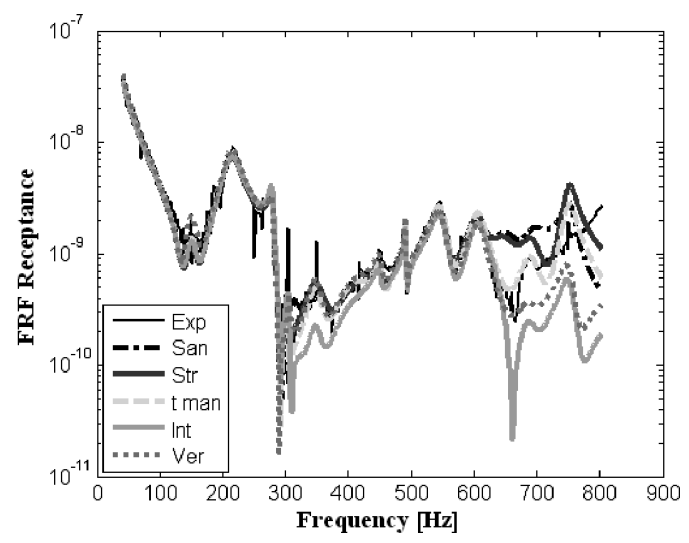

(d)

Fig. 4 Curve fit using all weighting function for measurement point number 20 (a, b) and number 7 (c, d)
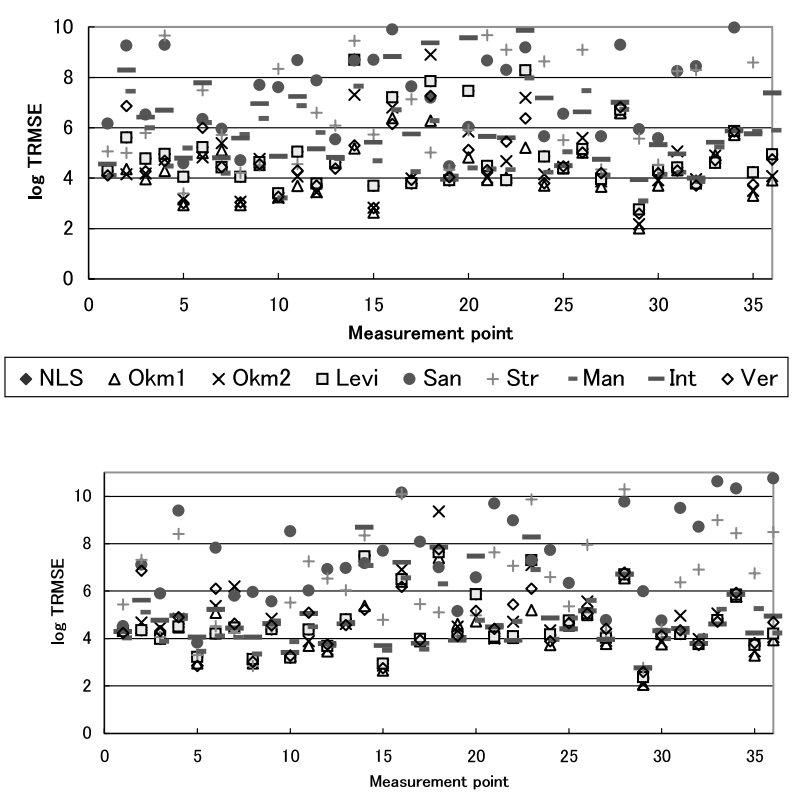

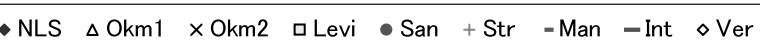

Fig. 5 Total RMSE in all measurement point using original weighting function (above) and frequency scale weighting function (below) for the second case tion with high total RMSE in most of measurement points. Okuma-1, Okuma-2 and Verboven are seen more consistent with relatively lower error, and others methods show their inconsistency.

Figure 6 shows the number of measurement points for which each weighting function gets the least RMSE. There is no very dominant weighting function except Okuma-1. CWLS contains some kinds of weighting functions, i.e. NLS, Okuma-1, Okuma-2, Levi, Stroble, t'Mannetje, and Verboven. In the case of using so many kinds of weighting functions, CWLS will consume much time. To solve this problem we recommend using the combination of weighting function which relative more consistent and lower error.

The weighting functions used for individual measurement points in CWLS are listed in Table 2. The result in this table can show that frequency scaling of weighting function does not improve curve fitting significantly.

\subsection{Section summary}

According to the abovementioned two case studies, it can be seen that different kinds of structures and the different measurement points in a structure have different noises and dynamic characteristics, and that modal curve fitting 

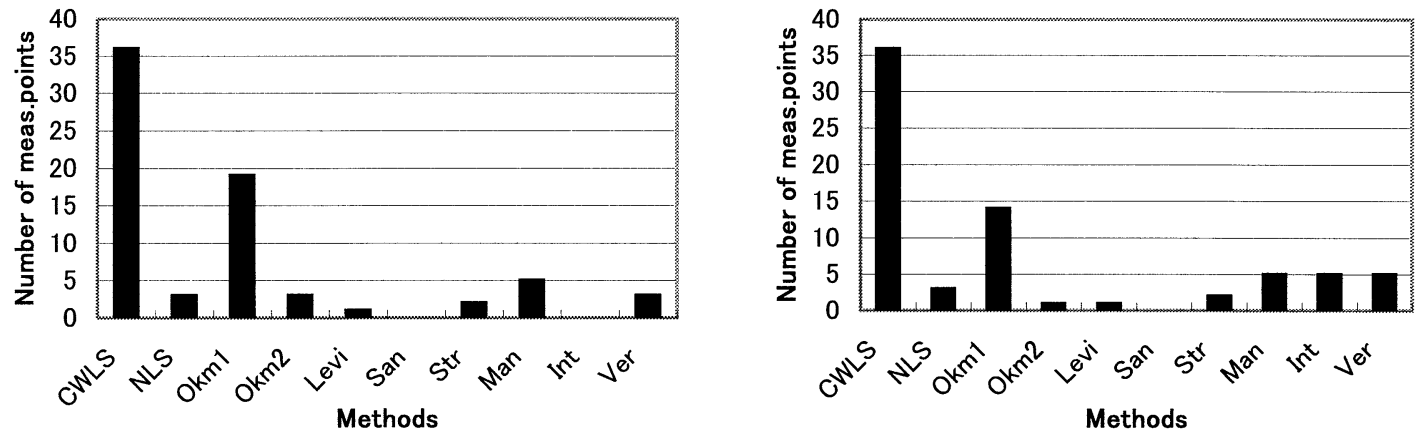

Fig. 6 The number of measurement points for which each weighting function gets the least RMSE, original weighting function (left) and weighting function with frequency scale (right)

method using a single kind of weighting function for FRFs of all measurement points cannot get good result. Moreover, curve fittings method using some kind of weighting function show inconsistency in their result. In addition, as the result, CWLS can provide better fitting results than conventional ones.

\section{Conclusion}

In this paper, nine kinds of weighting functions are applied to the least square method in order to obtain better curve fitting of FRFs of two actual mechanical systems. The result indicates the inconsistence of some weighting function in all measured FRFs.

The application of combination of weighted least square can overcome the inconsistence problem of the implementation of single weighting function in modal curve fitting. This proposed method give more accurate model by minimizing error in each FRF of measurement point.

However, investigating all weighting function in each measurement point makes combination of weighted least square becomes inefficient. Furthermore, if the short time computation is needed, it is recommended to use only Okuma-1, or its combination with Okuma-2, and Verboven in all condition for weighted least square, because those weighting function are relative more consistent and have lower error than the others.

\section{References}

( 1 ) Allemang, R.J. and Brown, D.L., A Unified Matrix Polynomial Approach to Modal Identification, Journal of Sound and Vibration, Vol.211, No.3 (1998), pp.301322.

( 2 ) Maia, S., He, L., Lin, S. and To, U., Theoretical and Experimental Modal Analysis, (1997), Chap.4, Research Studies Press Ltd., Hertfordshire, England.

( 3 ) Auweraer, H.V.D., Guillaume, P., Verboven, P. and Vanlanduit, S., Application of a Fast Stabilizing Fre- quency Domain Parameter Estimation Method, Journal of Dynamics Systems, Measurement, and Control, Vol.123 (2001), pp.651-658.

( 4 ) Pintelon, R., Guillaume, P., Rollain, Y., Schoukens, J. and Hamme, H.V, Parametric Identification of Transfer Function in the Frequency Domain-A Survey, IEEE Transaction on Automatic Control, Vol.39, No.11 (1994), pp.2245-2260.

( 5 ) Ewins, D.J., Modal Testing: Theory and Practice, (1984), Chap.4, Research Study Press Ltd., Hertfordshire, England.

(6) Okuma, M. and Nagamatsu, A., Experimental Identification of a Mechanical Structure with Characteristic Matrix (7th Report, Proposition of a Practical and Useful Method), Journal of JSME-D, (in Japanese), Vol.54, No.507 (1988), pp.2557-2564.

( 7 ) Okuma, M., Yamaguchi, M. and Nagamatsu, A., Practical and Realistic Weighting Function for Modal Curve Fitting, The ASME PVP Conference, Vol.179 (1989), pp.57-77.

( 8 ) Levi, E.C, Complex Curve Fitting, IEEE Transaction of Automatic Control, Vol.AC-4 (1959), pp.37-44.

(9) Sanathanan, C.K. and Koerner, J., Transfer Function Synthesis as a Ratio of Two Complex Polynomials, IEEE Transaction of Automatic Control, Vol.AC-9, No.1 (1963), pp.56-58.

(10) Whitfield, A.H., Transfer Function Synthesis Using Frequency Response Data, International Journal of Control, Vol.43, No.5 (1986), pp.1413-1426.

(11) Verboven, P., Guillaume, P., Chauberghe, B., Vanlaundit, S. and Parlo, E., A Comparison of Frequency-Domain Transfer Function Model Estimator Formulations for Structural Dynamics Modeling, Journal of Sound and Vibration, Vol.279, No.3 (2005), pp.775-798.

(12) Okuma, M., Shi, Q. and Oho, T., Development of the Experimental Spatial Matrix Identification Method (Theory and Basic Verification with a Frame Structure), Journal of Sound and Vibration, Vol.219, No.1 (1999), pp.5-22. 